\title{
Psicooncología
}

ISSN: $1696-7240$

\section{Validación del cuestionario básico de síntomas M. D. Anderson(MDASI) para pacientes con cáncer en población mexicana}

\author{
Oscar Galindo Vázquez ${ }^{1 *}$; Cristina Noemi Aguilar González²; Abelardo Meneses García \\ German Calderillo Ruiz"; Enrique Bargalló Rocha ${ }^{5}$; Ángel Herrera Gómez ${ }^{6}$
}

Recibido: 6 de marzo de 2020 / Aceptado: 25 de abril de 2021

Resumen. Introducción: Los diferentes síntomas en el paciente con cáncer pueden afectar las actividades en la vida cotidiana, las decisiones terapéuticas, así como la calidad de vida. Por lo que identificar la sintomatología en este grupo de pacientes es de suma importancia. Objetivo: Validar el cuestionario básico de síntomas M. D. Anderson (MDASI) para pacientes con cáncer en población mexicana. Método: Se incluyeron 309 pacientes del Instituto Nacional de Cancerología con edad entre 18 a 85 años, el tipo de estudio fue transversal, no experimental. Instrumentos: Cuestionario básico de síntomas MDASI, Cuestionario de Calidad de Vida de la Organización Europea para el tratamiento e Investigación del cáncer (EORTC-QLQ-C30) y Termómetro de Distrés. Resultados: Un análisis factorial ajustado a dos factores presentó un instrumento con 12 reactivos, similar a la versión original. La consistencia interna de la escala global mostró un índice satisfactorio $(0,86)$. Las alfas de Cronbach de cada subescala tuvieron un valor de 0,79 y 0,80 que explicaron el 48,04\% de la varianza global. La validez, por medio de correlación con las medidas concurrentes, mostró resultados significativos ( $\mathrm{r}$ de Pearson de $0,954 \mathrm{a}-0,267, \mathrm{p}<0,01$ ). Conclusiones: El instrumento MDASI mostro propiedades psicométricas adecuadas, similar a la versión original. Es útil para la práctica clínica e investigación. Se recomienda realizar estudios complementarios para determinar su sensibilidad en efectos de intervenciones médicas y psicológicas.

Palabras clave: Cáncer, población mexicana, cuestionario, síntomas, validación psicométrica.

\section{[en] Validation of the basic symptom questionnaire M.D. Anderson (MDASI-M) for cancer patients in the Mexican population}

Abstract. Introduction: The different symptoms in the patient with cancer can affect the activities in the daily life, the therapeutic decisions, as well as the quality of life. Thus, identifying the symptomatology

1 Servicio de psicooncología, Instituto Nacional de Cancerología. INCan. México.

E-mail: psigalindo@yahoo.com.mx

2 Servicio de psicooncología, Instituto Nacional de Cancerología. INCan. México.

E-mail: christina.aguilar1@hotmail.com

3 Dirección General, Instituto Nacional de Cancerología. INCan. México.

E-mail: amenesesg@incan.edu.mx

4 Subdirección de Medicina Interna, Instituto Nacional de Cancerología. INCan. México.

E-mail: medicinainterna.incan2018@gmail.com

5 Clínica de cáncer de mama, Instituto Nacional de Cancerología. INCan. México.

E-mail: ebargallo@incan.edu.mx

6 Dirección general adjunta médica, Instituto Nacional de Cancerología. INCan. México.

E-mail: aherreragc@incan.edu.mx

* Dirección de correspondencia: Dr. Oscar Galindo Vázquez. Servicio de Psicooncología, Instituto Nacional de Cancerología, Calle Av. San Fernando No.22, Colonia. Sección XVI Ciudad de México, C.P. 14080.

E-mail: psigalindo@yahoo.com.mx 
in this group of patients is of the utmost importance. Objective: To validate the basic symptoms questionnaire M. D. Anderson (MDASI) for cancer patients in the Mexican population. Method: 309 patients from the National Cancer Institute were included, aged between 18 and 85 years, the type of study was transversal, not experimental. Instruments: Basic questionnaire of symptoms MDASI, Inventory of Quality of Life of the European Organization for the treatment and researching of cancer (EORTC-QLQ-C30) and Thermometer of Distrés. Results: A factorial analysis adjusted to two factors showed an instrument with 12 items, similar to the original version. The internal consistency of the global scale presented a satisfactory index (0.86). The Cronbach alphas of each subscale had a value of 0.79 and 0.80 that explained $48.04 \%$ of the global variance. The validity, by means of correlation with the concurrent measures, showed significant results (Pearson's $r$ from 0.954 to $-0.267, p<0.01$ ). Conclusions: The MDASI instrument produced adequate psychometric properties, similar to the original version. It is useful for clinical practice and research. Complementary studies are recommended to determine their sensitivity in the effects of medical and psychological interventions.

Keywords: Cancer, Mexican population, psychometric validation, questionnaire, symptom.

Sumario. 1. Introducción 2. Método 3. Resultados 4. Discusión 5. Conclusiones 6. Referencias

Como citar: Galindo Vázquez O, Aguilar González CN, Meneses García A, Calderillo Ruiz G, Bargalló Rocha E, Herrera Gómez A. Validación del cuestionario básico de síntomas M. D. Anderson (MDASI) para pacientes con cáncer en población mexicana. Psicooncología 2021; 18: 347-358, https:// dx.doi.org/10.5209/psic. 77757

\section{Introducción}

El manejo de síntomas físicos y emocionales en los pacientes con cáncer representan unos de los principales objetivos de atención por parte del equipo de salud. La prevalencia de síntomas como fatiga, dolor, debilidad, tristeza, ansiedad, disnea, vómito y deterioro cognitivo han sido consistentemente reportados en pacientes oncológicos, con un promedio de 10 síntomas durante el periodo de tratamiento y cuatro durante los primeros meses posteriores a este ${ }^{(1)}$. Si se manejan de manera deficiente, estos síntomas pueden tener un impacto considerable en la capacidad de funcionamiento de los pacientes, la calidad de vida, la capacidad para cumplir con los tratamientos contra el cáncer y el uso de los recursos de atención médica ${ }^{(2)}$ la evidencia sobre las asociaciones entre los síntomas y el estadio en el momento del diagnóstico puede ayudar a orientar el diseño de intervenciones de diagnóstico temprano ${ }^{(3)}$.

En este contexto la asociación de los diferentes síntomas, es un problema frecuente, por ejemplo, la fatiga se reporta como el síntoma más preocupante a causa de su alta prevalencia ${ }^{(4)}$ la mayoría de los pacientes informan sentirse fatigados debido a le tratamiento contra el cáncer para realizar actividades físicas ${ }^{(5)}$. Estos síntomas están asociados con problemas psicológicos principalmente depresión ${ }^{(6)}$. Pueden ser causados por la enfermedad misma y por la combinación de los tratamientos (quimioterapia, radioterapia y/o cirugía). Otros mecanismos asociados son trastornos del sueño, condiciones medioambientales, niveles de actividad y estatus nutricional ${ }^{(7,8)}$.

Teunissen et al. ${ }^{(9)}$ en una revisión sistemática de la literatura con 44 estudios (25,074 pacientes) identificaron 37 síntomas evaluados. Casi todos los síntomas se produjeron en más del $10 \%$ de los pacientes. Cinco síntomas (fatiga, dolor, falta de 
energía, debilidad y pérdida de apetito) se produjeron en más de $50 \%$. Los pacientes más jóvenes tenían más probabilidades de informar síntomas de dolor y náuseas moderados a graves, las mujeres tenían más probabilidades de informar síntomas de ansiedad / depresión de moderados a graves ${ }^{(10)}$. Estos síntomas son el resultado del cáncer y su tratamiento, los cuales incluso pueden persistir años después de terminado el mismo ${ }^{(11)}$; lo que constituye una enfermedad muy estresante para los pacientes y para sus cuidadores primarios informales ${ }^{(12)}$.

Por lo que evaluar los síntomas de forma oportuna y concurrentemente al tratamiento es necesario para valorar íntegramente la efectividad del mismo ${ }^{(1)}$. En este sentido es necesario emplear instrumentos válidos y confiables en pacientes ambulatorios y hospitalizados, que permitan determinar los efectos y beneficios de los tratamientos ${ }^{(13)}$ Así como las principales necesidades a nivel de sintomatología física y psicológica entre diferentes grupos de pacientes.

En este contexto, el Cuestionario Básico de Síntomas M. D. Anderson (MDASI) desarrollado por Cleeland et al. ${ }^{(1)}$ es una escala auto-aplicada conformada por 19 ítems y dos áreas: intensidad de síntomas e interferencia en la vida cotidiana durante las últimas 24 horas. Es una escala que se puede contestar en 10 minutos, durante el periodo de tratamiento y seguimiento en cualquier proceso oncológico.

La escala original ha sido adaptada y validada en diversas culturas mostrando una adecuada consistencia interna (alfas entre 0,75 y 0,93 ), y validez convergente $(0,80)$, así como una estructura factorial congruente con lo reportado en la versión original desarrollada por Cleeland et al. ${ }^{(1)}$.

Aunque en México hay instrumentos validados en población mexicana diseñados para evaluar calidad de vida (EORTC QLQ-30 y FACT) su objetivo específico no es evaluar sintomatología y su afectación en la vida cotidiana del paciente oncológico por lo que el presente estudio tuvo el objetivo de validar el Cuestionario Básico de Síntomas M. D. Anderson (MDASI) en una muestra de pacientes con cáncer en población mexicana.

\section{Método}

Se incluyeron a 309 pacientes oncológicos de ambos sexos (Ver tabla 1). La obtención de la muestra fue por disponibilidad en el Instituto Nacional de Cancerología (INCan). La muestra de participantes se obtuvo durante las consultas de los Servicios de Oncología Médica, Cirugía, Radioterapia y Psico-Oncología. El proyecto fue aprobado por el comité científico y de ética en investigación del INCan, cada uno de los participantes acepto participar en el presente estudio firmando el consentimiento informado de investigación.

Los criterios de inclusión fueron: (a) cualquier tipo de diagnóstico oncológico, (b) en tratamiento oncológico (cualquier tipo o combinación de estos), (c) cualquier estadio, (d) que sepan leer y escribir. Como criterios de exclusión se consideró: (a) pacientes que presenten cuadros psiquiátricos severos $\mathrm{y} / \mathrm{o}$ adicción a alguna sustancia psicoactiva, (b) pacientes en crisis psicológica y (c) pacientes con deficiencias cognitivas, auditivas y/o visuales severas. 
Tabla 1. Descripción de la muestra de validación del MDASI-M

\begin{tabular}{|c|c|c|c|c|c|}
\hline & $\mathbf{f}$ & $\%$ & & $\mathbf{f}$ & $\%$ \\
\hline $\mathrm{N}$ & 309 & 100 & & & \\
\hline \multirow[t]{2}{*}{ Edad (rango) 18-85 años } & & & Diagnóstico & & \\
\hline & & & Cáncer de mama & 112 & 36,2 \\
\hline Genero & & & Cabeza y cuello & 36 & 11,7 \\
\hline Masculino & 121 & 39,2 & Urológico & 70 & 22,7 \\
\hline \multirow[t]{2}{*}{ Femenino } & 188 & 60,8 & Gástricos & 12 & 3,9 \\
\hline & & & Ginecológicos & 25 & 8,1 \\
\hline Escolaridad & & & Neumología & 23 & 7,4 \\
\hline Ninguna & 20 & 6,5 & Otros & 31 & 10,0 \\
\hline Primaria & 79 & 25,6 & & & \\
\hline Secundaria & 76 & 24,6 & Estadio & & \\
\hline Bachillerato & 74 & 23,9 & I & 34 & 11,0 \\
\hline Licenciatura & 50 & 16,2 & II & 97 & 31,0 \\
\hline \multirow[t]{2}{*}{ Posgrado } & 10 & 3,2 & III & 100 & 32,4 \\
\hline & & & IV & 78 & 25,3 \\
\hline Estado civil & & & Tratamiento & & \\
\hline Soltero(a) & 57 & 18,4 & Quimioterapia & 154 & 49,8 \\
\hline Casado(a)/Juntado & 181 & 58,6 & Cirugía & 39 & 12,6 \\
\hline Divorciado(a) & 48 & 15,5 & Radio/Quimio & 57 & 18,4 \\
\hline \multirow[t]{2}{*}{ Viudo(a) } & 23 & 7,4 & Radioterapia & 31 & 10,0 \\
\hline & & & Otros & 28 & 9,2 \\
\hline \multicolumn{6}{|l|}{ Paternidad } \\
\hline $\mathrm{Si}$ & 246 & 79,6 & Comorbilidad & & \\
\hline \multirow[t]{2}{*}{ No } & 63 & 20,4 & $\mathrm{Si}$ & 108 & 35,0 \\
\hline & & & No & 201 & 65,0 \\
\hline \multicolumn{6}{|l|}{ Nivel socioeconómico } \\
\hline 0 & 139 & 45,0 & Índice Karnofsky & & \\
\hline 1 & 65 & 21,0 & 60 & 5 & 1,6 \\
\hline 2 & 66 & 21,4 & 70 & 10 & 3,2 \\
\hline 3 & 30 & 9,7 & 80 & 47 & 15,2 \\
\hline \multirow[t]{2}{*}{4} & 9 & 2,9 & 90 & 174 & 56,3 \\
\hline & & & 100 & 73 & 23,6 \\
\hline
\end{tabular}

\section{Adaptación al español}

Inicialmente se solicitó la autorización del Departamento de Investigación en Síntomas del University of Texas MD Anderson Cancer Center, para validarlo en población mexicana. El objetivo 1 adaptación en población mexicana, se realizó mediante una prueba piloto en 30 pacientes mexicanos de la versión en castellano del MDASI. Se aplicó un formato de registro de caso que incluyo un cuestionario específico sobre las instrucciones, sobre cada reactivo y las opciones de respuesta. Estas preguntas tuvieron el objetivo de evaluar si los pacientes comprendían el ítem 
y si existía alguna expresión confusa u ofensiva. Se registraron las dificultades, se realizó una revisión sistemática de éstas y se hicieron las modificaciones pertinentes.

Posteriormente se aplicó concurrentemente el Cuestionario Básico de Síntomas M. D. Anderson (MDASI) desarrollado por Cleeland et al. (2000)(1), adaptado a población mexicana, el Inventario de Calidad de Vida (EORTC QLQ-C30) Aaronson et al. (1993) y el Termómetro de Distrés. Se incluyó una cédula de identificación del participante en la cual se recabaron variables sociodemográficas y clínicas (edad, sexo, estado civil, escolaridad, tipo de diagnóstico, estadio de la enfermedad, tratamiento actual y/o periodo de seguimiento, peso, ECOG y otras enfermedades médicas co-mórbidas).

\section{Instrumentos}

Cuestionario básico de síntomas M. D. Anderson-MDASI -

El MDASI- fue desarrollado por Cleeland et al. ${ }^{(14)}$, se trata de una escala autoaplicada conformada por dos áreas: intensidad de síntomas (13 reactivos) e interferencia en la vida cotidiana (6 reactivos) con un formato de respuesta que va de 0 (No estuvo presente) a 10 (El peor que pueda imaginar) y 0 (No han interferido) a 10 (Interfirieron totalmente). Estos permiten evaluar la gravedad de diferentes síntomas y el impacto en la vida cotidiana del paciente oncológico en diferentes tipos de tratamiento durante las últimas 24 horas. Su aplicación se lleva a cabo en un tiempo de 10 minutos.

Inventario de Calidad de Vida de la Organización Europea para el tratamiento e Investigación del cáncer-EORTC-QLQ-C30

El EORTC QLQ -C30- fue desarrollado por Aaronson et al. ${ }^{(4)}$ es un instrumento diseñado específicamente para pacientes con cáncer; en la actualidad ha sido evaluado y validado en estudios multinacionales y multiculturales. Validado en México Oñate et al. ${ }^{(15)}$ Este instrumento es multidimensional, ya que evalúa cinco escalas funcionales (abarcando las áreas físicas, de desempeño, cognitiva, emocional y social), tres escalas sintomáticas (fatiga, dolor y náusea/vómito), una escala global de calidad de vida y otros síntomas que los pacientes presentan frecuentemente. Está diseñado como instrumento auto-aplicable, es decir, el paciente lo puede llenar en alrededor de 10 minutos. Las alfas de Cronbach de las escalas van de 0,32 a 0,90 . Como validez concurrente se obtuvieron correlaciones estadísticamente significativas ( $r$ de Pearson de 0,37 a $0,43, p<0,05$ ) entre el EORTC QLQ30 y los niveles de albumina.

\section{Termómetro de Distrés}

Termómetro de Distres desarrollado por Holland y Anderson ${ }^{(16)}$ fue validado para población mexicana por Almanza-Muñoz et al. ${ }^{(17)}$ el termómetro de distrés consta de dos partes. La primera es una escala análoga visual, ubicada en forma vertical y bajo la apariencia de un termómetro. Las instrucciones son: "encierre en un círculo (0 al 10) que mejor describa que tanto distrés ha experimentado en la 
última semana, incluyendo el día de hoy". En la segunda se le pide al sujeto que, de una lista de 35 problemas físicos y emocionales, señale la existencia o no de los mismos. Las propiedades psicométricas del termómetro de distrés en población mexicana muestran que la confiabilidad por consistencia interna obtenida fue de alfa de Cronbach $=0,87$. La validación convergente a través del Análisis ROC mostró un área bajo la curva de 0,63 , sensibilidad de $93 \%$, especificidad de $76 \%$, valor predictivo positivo de $82 \%$, valor predictivo negativo de $90 \%$, con un punto de corte de cuatro o más, lo cual arroja una prevalencia del distrés de $61,8 \%$.

\section{Análisis estadísticos}

Se empleó un análisis factorial de componentes principales con rotación oblimin directo para determinar validez de constructo. Los criterios utilizados para el análisis factorial fueron: (a) cargas factoriales $\geq 0,40$ y (b) coeficiente de consistencia interna por cada factor alfa de Cronbach $\geq 0,60$. La validez concurrente mediante el coeficiente de correlación de Pearson entre las medidas del MDASI, el EORTCQLQ30, índice Karnofsky y el termómetro de distrés. El valor alfa establecido fue de 0,05 .

\section{Resultados}

\section{Análisis factorial}

Se obtuvo en la prueba de esfericidad de Bartlett $(p=0,001)$ y un índice de adecuación muestral $(\mathrm{KMO}=0,922)$ que determinó la suficiencia de la muestra para la estructura de los componentes finales. El análisis factorial se realizó mediante método de componentes principales y rotación Oblimin en el área intensidad de síntomas con 13 reactivos arrojó un modelo con dos factores que explico el $62,15 \%$ de la varianza. Ningún reactivo fue eliminado y los resultados concuerdan con la estructura factorial de la versión original (Ver Tabla 2).

Tabla 2 . Análisis factorial MDASI-M

\begin{tabular}{lccc}
\hline $\begin{array}{l}\text { Escala Global } \alpha=, 94 \\
\text { Varianza Explicada }=64,39 \%\end{array}$ & $\begin{array}{c}\text { Factor } \\
\text { I }\end{array}$ & $\begin{array}{c}\text { Factor } \\
\text { II }\end{array}$ & $\begin{array}{c}\text { Alfa de } \\
\text { Cronbach }\end{array}$ \\
\hline AREA 1 INTENSIDAD DE SíNTOMAS & & & \\
& & & $\alpha=, 92$ \\
1. Dolor, & $\mathbf{7 1 4}$ &, 534 & \\
2. Fatiga, & $\mathbf{7 9 1}$ &, 678 & \\
3. Nausea, &, 662 & $\mathbf{8 6 8}$ & \\
4. Desvelo, & $\mathbf{, 7 9 0}$ &, 664 & \\
5. Sufrimiento emocional, & $\mathbf{8 2 0}$ &, 577 & \\
\hline
\end{tabular}




\begin{tabular}{|c|c|c|c|}
\hline $\begin{array}{l}\text { Escala Global } \alpha=, 94 \\
\text { Varianza Explicada }=64,39 \%\end{array}$ & $\begin{array}{c}\text { Factor } \\
\text { I }\end{array}$ & $\begin{array}{c}\text { Factor } \\
\text { II }\end{array}$ & $\begin{array}{c}\text { Alfa de } \\
\text { Cronbach }\end{array}$ \\
\hline 6. Falta de aire, & ,688 & ,653 & \\
\hline 7. Dificultad para recordar cosas, &, 522 & ,618 & \\
\hline 8. Falta de apetito, & ,692 & ,829 & \\
\hline 9. Somnolencia, & ,797 & ,709 & \\
\hline 10. Sequedad bucal & ,775 & ,718 & \\
\hline 11. Tristeza & ,799 &, 551 & \\
\hline 12. Vómito &, 542 & ,812 & \\
\hline 13. Entumecimiento u hormigueo & ,754 & 639 & \\
\hline Alfa de Cronbach ,82 & & & ,94 \\
\hline Varianza explicada 53,89\% & & & $8,26 \%$ \\
\hline \multicolumn{3}{|c|}{ AREA 2 INTERFERENCIA EN LA VIDA COTIDIANA } & $\alpha=, 90$ \\
\hline 14. Actividad en general, & ,695 & & \\
\hline 15.Estado de ánimo, & ,780 & & \\
\hline 16. Trabajo, & 690 & & \\
\hline 17. Relaciones con otras personas & ,641 & & \\
\hline 18. Capacidad para caminar, & 605 & & \\
\hline 19. Disfrutar la vida, & ,697 & & \\
\hline Varianza explicada & $49 \%$ & & \\
\hline
\end{tabular}

\section{Consistencia interna}

El alfa de Cronbach de la parte de intensidad de síntomas (13 reactivos) fue 0,92; se identificaron dos factores: Factor I (Síntomas generales formado por nueve reactivos con un $\alpha=0,82$ ) y Factor II (Síntomas gastrointestinales y dificultad para recordar, conformada por cuatro ítems con un $\alpha=0,94)$. En relación a la segunda parte interferencia en la vida cotidiana (6 reactivos) fue de 0,90; en tanto, el alfa de Cronbach de los 19 reactivos totales del MDASI fue 0,94 . La consistencia puede considerarse adecuada.

Validez externa por medio de correlaciones con medidas concurrentes

En la Tabla 3 se muestra el resumen de las asociaciones entre el MDASI, sus dos áreas, el EORTC-QLQ30, Índice Karnofsky y el Termómetro de Distrés. Como se puede observar las correlaciones fueron positivas o negativas dependiendo a la relación teórica entre subescalas y estadísticamente significativas ( $r$ de Pearson de 0,954 a $-0,267, p<0,01$ ) excepto con el ítem 16 (diarrea) del EORTC-QLQ30 (Ver Tabla 3). 
Tabla 3. Correlaciones entre MDASI total y por subescalas, subescalas del EORTC QLQ -C30 y Termómetro de Distrés

\begin{tabular}{|c|c|c|c|}
\hline & 1 & 2 & 3 \\
\hline 1. MDASI total & 1 & & \\
\hline 2. MDASI Intensidad & $0,954^{* *}$ & & \\
\hline 3. MDASI Interferencia & $0,846 * *$ & $0,648^{* *}$ & \\
\hline 4. EORTC Función física & $-0,514^{* *}$ & $-0,402^{* *}$ & $-0,592 * *$ \\
\hline 5. EORTC Rol funcional & $-0,457 * *$ & $-0,352 * *$ & $-0,535 * *$ \\
\hline 6. EORTC Función emocional & $-0,601^{* *}$ & $-0,546 * *$ & $-0,557 * *$ \\
\hline 7. EORTC Función cognitiva & $-0,430^{* *}$ & $-0,428^{* *}$ & $-0,334 * *$ \\
\hline 8. EORTC Función social & $-0,538 * *$ & $-0,435^{* *}$ & $-0,595 * *$ \\
\hline 9. EORTC Fatiga & $0,580 * *$ & $0,509 * *$ & $0,570 * *$ \\
\hline 10. EORTC Náusea y vómito & $0,395 * *$ & $0,393 * *$ & $0,306^{* *}$ \\
\hline 11. EORTC Dolor & $0,540 * *$ & $0,492 * *$ & $0,498 * *$ \\
\hline 12. EORTC Disnea & $0,410 * *$ & $0,373 * *$ & $0,379 * *$ \\
\hline 13. EORTC Insomnio & $0,450 * *$ & $0,425^{* *}$ & $0,387 * *$ \\
\hline 14. EORTC Apetito & $0,489 * *$ & $0,437 * *$ & $0,466^{* *}$ \\
\hline 15, EORTC Constipación & $0,321 * *$ & $0,298 * *$ & $0,286^{* *}$ \\
\hline 16. EORTC Diarrea & 0,69 & 0,58 & 0,75 \\
\hline 17. EORTC Dif. Financieras & $0,388 * *$ & $0,304 * *$ & $0,441^{*}$ \\
\hline 18. Termómetro de Distrés & $0,543 * *$ & $0,506 * *$ & $0,481 * *$ \\
\hline 19. Índice Karnofsky & $-0,267 * *$ & $-0,345^{* *}$ & $-0,323 * *$ \\
\hline
\end{tabular}

\section{Discusión}

La evaluación de los síntomas en pacientes oncológicos es de gran relevancia debido a que el equipo de salud y los pacientes se enfrentan a opciones de tratamientos que pueden ser igualmente eficaces, por lo que los síntomas reportados por el paciente se han convertido en una variable crítica en la toma de decisiones respecto al tratamiento. La medida de auto reporte de síntomas es fundamental para evaluar los efectos benéficos de los tratamientos farmacológicos, la toxicidad y la calidad de la supervivencia ${ }^{(11)}$.

De acuerdo con el presente estudio, el MDASI en el área de Intensidad de síntomas (13 reactivos) se identificaron dos Factores: Factor I Síntomas generales $(\alpha=0,82)$ y Factor II Síntomas gástricos y dificultad para recordar cosas $(\alpha=0,94)$. Como área general de intensidad de síntomas (factor I y factor II) presenta una adecuada consistencia interna $(\alpha=0,94)$.

Las características psicométricas del área intensidad de síntomas es similar con la versión original de Cleeland et al. ${ }^{(14)}$ quienes encontraron para el Factor I una consistencia interna de 0,85 y para el Factor II de 0,82. Así como con estudios previos 
en población asiática y caucásica con alfas de 0,80 a $0,92^{(11,18-20)}$. Otros estudios en población europea han identificado un solo factor en el área de intensidad de síntomas $(\alpha=0,78)^{(21)}$ o tres factores (síntomas generales, síntomas gastrointestinales y componentes afectivos y cognitivos) con alfas entre 0,69 y $0,79^{(20,21)}$.

En relación al área de Interferencia en la vida cotidiana en pacientes mexicanos, el MDASI mostró una adecuada consistencia interna $(\alpha=0,90)$ y una varianza explicada de $67,49 \%$ lo cual concuerda con la versión original de Cleeland et al. ${ }^{(14)}$ quienes reportan una consistencia interna de 0,94 . Otros estudios han identificado valores de 0,79 a $0,93^{(11,19,20,22-26)}$ lo cual indica adecuadas consistencias internas en poblaciones caucásicas y asiáticas.

La validez convergente mostró correlaciones medias y altas negativas con las subescalas del EORTC QLQ-C30 principalmente con las subescalas función social, emocional, física, cognitiva, social y rol funcional; medias y altas positivas con fatiga, nausea, dolor, disnea y pérdida de apetito; y con el Índice Karnofsky. Correlaciones identificadas en el mismo sentido y magnitud entre el MDASI y el EORTC QLQ-C30 fueron reportadas por otros estudios ${ }^{(20,24-26)}$ y con la encuesta de salud SF-36 ${ }^{(11,21,22)}$ lo cual respalda los resultados de este estudio. Por su parte la correlación entre el MDASI y el Índice Karnofsky ratificó una asociación con la medida de funcionalidad empleada por excelencia en oncología.

En cuanto a la validez convergente con constructos psicológicos, Okuyama et al. (25) correlacionaron el MDASI con la subescala de depresión del Perfil del Estado de Ánimo (POMS) obteniendo una correlación de $0,66(p<0,001)$; en el presente estudio, si bien el distrés (malestar emocional) no es equiparable con depresión sí muestra una correlación directa $(r=0,543, p<0,01)$, lo cual indica una validez convergente del MDASI con medidas psicológicas diversas.

En este contexto se requiere una detección oportuna e información precisa sobre la ocurrencia de estos efectos secundarios, así como asistencia de parte del equipo de salud para mitigar estos síntomas en la práctica habitual ${ }^{(2,27,28)}$. La evaluación mediante instrumentos válidos y confiables de las necesidades reportadas por las pacientes debe ser una parte integral de las intervenciones en oncología. Estas escalas deben estar relacionadas con la toma de decisiones sobre los tratamientos en conjunto con los equipos de salud multidisciplinarios ${ }^{(29)}$. Esto porque la mayoría de los pacientes con cáncer experimentan síntomas a lo largo de la trayectoria de la enfermedad, a menudo con mayor intensidad a medida que se acerca la muerte ${ }^{(10)}$ adicionalmente las familias también tienen un alto riesgo de sufrir morbilidad física y psicológica ${ }^{(30)}$.

En el mismo sentido el efecto en la interferencia en la vida cotidiana ha sido reportado en pacientes con cáncer con metástasis, una baja laboral incluso de cien por ciento y sólo el $13 \%$ de las pacientes sin metástasis presentan una actividad profesional. Estos datos reflejan las dificultades a las que se enfrentan los pacientes a nivel profesional: pueden experimentar problemas de discriminación laboral, despidos injustificados y limitaciones de responsabilidades laborales ${ }^{(31)}$. Por lo que intervenciones multidisciplinarias que coadyuven para el manejo de estos síntomas y que permitan en el paciente mayor funcionalidad a lo largo del tratamiento se muestran como necesarias en todo centro oncológico.

La validación del Cuestionario básico de síntomas M. D. Anderson (MDASI) en una muestra de pacientes mexicanos con cáncer resulta relevante porque no se han reportado la validaciones en poblaciones latinas, representa un instrumento 
de evaluación de sintomatología breve, fácil de aplicar y confiable para la práctica clínica y la investigación en población oncológica para determinar la magnitud de la intensidad de síntomas e interferencia en la vida del paciente, arribar a su prevención e instrumentar acciones para el tratamiento de los casos que lo ameriten, lo cual ayudara al equipo de salud y a los pacientes, en la toma de decisiones.

\section{Conclusiones}

La evaluación de los síntomas y la interferencia de estos en la vida cotidiana del paciente con cáncer es un aspecto relevante, más aún en población mexicana. En este contexto el instrumento MDASI mostro propiedades psicométricas adecuadas, similar a la versión original y a diferentes versiones validadas en otras poblaciones. De acuerdo a las propiedades psicométricas obtenidas se sugiere para práctica clínica e investigación, así como para identificar efectos de intervenciones en oncología y psicooncología.

\section{Referencias bibliográficas}

1. Cleeland CS. Symptom burden: multiple symptoms and their impact as patient-reported outcomes. J Natl Cancer Inst Monogr 2007;37:16-21. doi. https://doi.org/10.1093/ jncimonographs/lgm005

2. Henson LA, Maddocks M, Evans C, Davidson M, Hicks S, Higginson IJ. Palliative care and the management of common distressing symptoms in advanced cancer: Pain, breathlessness, nausea and vomiting, and fatigue. J Clin Oncol 2020; 38: 905. https://doi. org/10.1200/JCO.19.00470

3. Koo MM, Swann R, McPhail S, Abel GA, Elliss-Brookes L, Rubin GP, Lyratzopoulos G. Presenting symptoms of cancer and stage at diagnosis: evidence from a cross-sectional, population-based study. Lancet Oncol 2020;21:73-9. https://doi.org/10.1016/S14702045(19)30595-9

4. Aaronson NK, Ahmedzai S, Bergman B, Bullinger M, Cull A, Duez NJ. The European Organization for Research and Treatment of Cancer QLQ-C30: A quality-of-life instrument for use in international clinical trials in oncology. J Natl Cancer Inst 1993;85:365-76. https://doi.org/10.1093/jnci/85.5.365.

5. Frikkel J, Götte M, Beckmann M, Kasper S, Hense J, Teufel M, et al. Fatigue, barriers to physical activity and predictors for motivation to exercise in advanced Cancer patients. BMC palliative care 2020;19:1-11. https://doi.org/10.1186/s12904-020-00542-z

6. McFarland DC, Jutagir DR, Miller A, Nelson C. Physical problem list accompanying the distress thermometer: Its associations with psychological symptoms and survival in patients with metastatic lung cancer. Psychoncology 2020;29:910-9. https://doi.org/ 910919. https://doi.org/10.1002/pon.5367

7. Hinz A, Krauss O, Hauss JP, Hockel M, Kotmann RD, Stolzenburg JU, et al. Anxiety and depression in cancer patients compared with the general population. Eur J Cancer Care 2018;19:522-9. https://doi.org/10.1111/j.1365-2354.2009.01088.x

8. Pirl WF. Evidence report on the occurrence, assessment, and treatment of depression in cancer patients J Natl Cancer Inst Monogr 2004;22:32-39. https://doi.org/10.1093/ jncimonographs/lgh026 
9. Teunissen S, Wesker W, Kruitwagen C, de Haes H, Voest EE, de Graeff A. Review article symptom prevalence in patients with incurable cancer: a systematic review J Pain Symptom Manage 2007;34:94-104. https://doi.org/10.1016/j.jpainsymman.2006.10.015

10. Merchant SJ, Kong W, Brundage M, Booth CM. Symptom evolution in patients with esophageal and gastric cancer receiving palliative chemotherapy: a population-based study. Ann Surg Oncol 2021;28:79-87. https://doi.org/10.1245/s10434-020-09289-6

11. Wang XS, Cleeland CS, Mendoza TR, Yun YH, Wang Y, Okuyama T, et al. Impact of cultural and linguistic factors on symptom reporting by patients with cancer. $\mathrm{J}$ Natl Cancer Inst 2010;102:732-8. https://doi.org/10.1093/jnci/djq097

12. Ortega-Andeane P, Galindo Vázquez O, Meneses García A. Factores ambientales y estrés en salas de espera: comparación en cuidadores primarios informales de pacientes con Cáncer. Rev Latin Med Cond/Latin Am J Behav Med 2018;9:41-50.

13. Kleiboer A, Gowing K, Hansen H, Hibberd C, Hodges L, Walker J, et al. Monitoring symptoms at home: what methods would cancer patients be comfortable using? Qual Life Res 2010;19: 965-8. https://doi.org/10.1007/s11136-010-9662-0

14. Cleeland CS, Mendoza TR, Wang XS, Chou C, Harle MT, Morrissey M, et al. Assessing symptom distress in cancer patients: the MD Anderson Symptom Inventory. Cancer 2000;89:1634-46.

15. Onate-Ocana LF, Alcántara-Pilar A, Vilar-Compte D, García-Hubard G, Rojas-Castillo E, Alvarado-Aguilar S, et al. Validation of the Mexican Spanish version of the EORTC C30 and STO22 questionnaires for the evaluation of health-related quality of life in patients with gastric cancer. Ann Sur Oncol 2009;16:88-95. https://doi.org/10.1245/s10434-008-0175-9

16. Holland JC, Anderson B. NCCN Guidelines for distress management. JNCCN 2007;5:66-98.

17. Almanza-Muñoz JDJ, Juárez IR, Pérez S. Traducción, adaptación y validación del Termómetro de Distrés en una muestra de pacientes mexicanos con cáncer. Revista de Sanidad Militar 2008;62:209-17.

18. Yun YH, Mendoza TR, Kang IO, You CH, Roh JW, Lee CG, et al. Validation study of the Korean version of the MD Anderson Symptom Inventory. J Pain Symptom Manage 2006;31:345-52. https://doi.org/10.1016/j.jpainsymman.2005.07.013

19. Mendoza T, Sheshadri A, Altan M, Hess K, George G, Stephen B, et al. Evaluating the psychometric properties of the immunotherapy module of the MD anderson symptom inventory. J immunother cancer 2020;8(2): e000931. https://doi.org/10.1136/jitc-2020000931

20. Schmidt H, Cleeland CS, Bauer A, Landenberger M, Jahn P. Symptom burden of cancer patients: Validation of the German MD Anderson Symptom Inventory. A cross-sectional multicenter study. J Pain Symptom Manage 2014; 49:117-25. https://doi.org/10.1016/j. jpainsymman.2014.04.007

21. Ivanova MO, Ionova TI, Kalyadina SA, Uspenskaya OS, Kishtovich AV, Guo H, et al. Cancer-related symptom assessment in Russia: validation and utility of the Russian MD Anderson Symptom Inventory. J Pain Symptom Manage 2005;30: 443-53. https://doi. org/10.1016/j.jpainsymman.2005.04.015

22. Lin CC, Chang AP, Cleeland CS, Mendoza TR, Wang XS. Taiwanese version of the MD Anderson symptom inventory: symptom assessment in cancer patients. J Pain Symptom Manage 2007;33:180-8. https://doi.org/10.1016/j.jpainsymman.2006.07.018

23. Nejmi M, Wang XS, Mendoza TR, Gning I, Cleeland CS. Validation and application of the Arabic version of the MD Anderson symptom inventory in Moroccan patients with cancer. J Pain Symptom Manage 2010;40:75-86. https://doi.org/10.1016/j. jpainsymman.2009.12.007 
24. Guirimand F, Buyck JF, Lauwers-Allot E, Revnik J, Kerguen T, Aegerter P, et al. Cancer-related symptom assessment in France: validation of the French MD Anderson Symptom Inventory. J Pain Symptom Manage 2010;39:721-33. https://doi.org/10.1016/j. jpainsymman.2009.08.014

25. Okuyama T, Wang XS, Akechi T, Mendoza TR, Hosaka T, Cleeland CS, et al. Japanese version of the MD Anderson Symptom Inventory: a validation study. J Pain Symptom Manage 2003;26:1093-104. https://doi.org/10.1016/j.jpainsymman.2003.05.003

26. Yun YH, Mendoza TR, Kang IO, You CH, Roh JW, Lee CG, et al. Validation study of the Korean version of the MD Anderson Symptom Inventory. J Pain Symptom Manage 2006; 31: 345-52. https://doi.org/10.1016/j.jpainsymman.2005.07.013

27. Brezden CB, Phillips KA, Abdolell M, Bunston T, Tannock IF. Cognitive Function in breast cancer patients receiving adjuvant chemotherapy. J Clin Oncol 2000; 18:2695-701. https://doi.org/10.1200/JCO.2000.18.14.2695

28. Jansen CE, Miaskowski C, Dodd M, Dowling G, Kramer J. A metaanalysis of studies of the effects of cancer chemotherapy on various domains of cognitive function. Cancer 2005;104:2222-33. https://doi.org/10.1002/cncr.21469

29. Vázquez ÓG, Menes MC, Ortiz DI, Muñoz SAB, Talamantes AL, García AM. Propiedades psicométricas del Instrumento de Evaluación de Funcionalidad en el Tratamiento para el Cáncer Cervicouterino (FACT-Cx) en pacientes mexicanas. Psicología y Salud 2021;31: 155-64.

30. Kross EK, Pollak KI, Curtis JR. Addressing the psychological symptoms of critical illness: the importance of "negative" trials in guiding next steps. JAMA 2019; 321:7. 649-50. https://doi.org/10.1001/jama.2019.0072

31. Font Guitera A, Reventós Osuna P, Falo Zamora C. Afrontamiento y calidad de vida en pacientes con cáncer de mama: Estudio preliminar de semejanzas y diferencias entre pacientes con y sin metástasis. Psicooncologia 2020;17: 179-94. https://doi.org/10.5209/ psic. 68249 